\title{
OPEN Transcranial magnetic stimulation entrains alpha oscillatory activity in occipital cortex
}

\author{
Yong-Jun Lin ${ }^{1 \bowtie}$, Lavanya Shukla², Laura Duguéé, ${ }^{1,2,6,7}$, Antoni Valero-Cabré ${ }^{3,4,5,8} \&$
} Marisa Carrasco ${ }^{1,2,8}$

Parieto-occipital alpha rhythms (8-12 Hz) underlie cortical excitability and influence visual performance. Whether the synchrony of intrinsic alpha rhythms in the occipital cortex can be entrained by transcranial magnetic stimulation (TMS) is an open question. We applied 4-pulse, $10-\mathrm{Hz}$ rhythmic TMS to entrain intrinsic alpha oscillators targeting right V1/V2, and tested four predictions with concurrent electroencephalogram (EEG): (1) progressive enhancement of entrainment across time windows, (2) output frequency specificity, (3) dependence on the intrinsic oscillation phase, and (4) input frequency specificity to individual alpha frequency (IAF) in the neural signatures. Two control conditions with an equal number of pulses and duration were arrhythmic-active and rhythmic-sham stimulation. The results confirmed the first three predictions. Rhythmic TMS bursts significantly entrained local neural activity. Near the stimulation site, evoked oscillation amplitude and intertrial phase coherence (ITPC) were increased for 2 and 3 cycles, respectively, after the last TMS pulse. Critically, ITPC following entrainment positively correlated with IAF rather than with the degree of similarity between IAF and the input frequency $(10 \mathrm{~Hz})$. Thus, we entrained alpha-band activity in occipital cortex for $\sim 3$ cycles $(\sim 300 \mathrm{~ms})$, and IAF predicts the strength of entrained occipital alpha phase synchrony indexed by ITPC.

Brain oscillations play an important functional role in perception, attention and cognition ${ }^{1-3}$. Specifically, alphaband $(8-12 \mathrm{~Hz})$ neural oscillations reflect ongoing sensory processing. Alpha power inversely correlates with excitability in vision ${ }^{4-9}$, audition ${ }^{10}$, and somatosensation ${ }^{11}$. In the visual cortex, the parieto-occipital alphaband phase correlates with baseline cortical excitability (phosphene threshold) ${ }^{12}$, gamma-band $(40-100 \mathrm{~Hz})$ power $^{13}$, and spiking responses ${ }^{14}$. In V1, alpha and gamma rhythms index feedback and feedforward processing, respectively ${ }^{15,16}$. Theories of oscillations postulate that alpha power reflects an active inhibition of task-irrelevant sensory signals ${ }^{17-19}$ whereas alpha phase reflects pulsed inhibition ${ }^{17,20}$, cyclic perceptual sampling ${ }^{2}$ or temporal expectation ${ }^{21}$. Here we aimed to use transcranial magnetic stimulation (TMS) in each trial to directly manipulate local alpha rhythms while we assess its neurophysiological impact with concurrent EEG.

The topography of alpha power is altered by spatial attention. Typically, in endogenous (voluntary) spatial attention tasks, alpha power contralateral to the cued visual field shows reduced alpha power while the ipsilateral side shows increased alpha power ${ }^{22-29}$. Moreover, this lateralized alpha power modulation could reach $V 1^{29,30}$. The parieto-occipital alpha-band activity is linked with the allocation of covert spatial attention and receives feedback control from frontoparietal cortices. The disruption of anticipatory pre-stimulus alpha rhythms on the occipital cortex brought about by transcranial magnetic stimulation (TMS) on the right intraparietal sulcus (IPS) or right frontal eye field (FEF) has been associated with deteriorated visual performance ${ }^{31-33}$. With Granger causality analyses applied to magnetoencephalogram data, alpha power modulation in the right FEF has been reported to predict alpha activity in V1, indicating feedback control ${ }^{34}$. Thus, V1/V2 may receive feedback control from FEF and IPS, two crucial nodes of the dorsal attention network.

\footnotetext{
${ }^{1}$ Department of Psychology, New York University, New York, NY 10003, USA. ${ }^{2}$ Center for Neuroscience, New York University, New York, NY 10003, USA. ${ }^{3}$ Cerebral Dynamics, Plasticity and Rehabilitation Group, Frontlab Team, Institut du Cerveau et de la Moelle, CNRS UMR 7225, INSERM U 1127 and Sorbonne Université, Paris, France. ${ }^{4}$ Department Anatomy and Neurobiology, Laboratory of Cerebral Dynamics, Boston University School of Medicine, Boston, USA. ${ }^{5}$ Cognitive Neuroscience and Information Tech. Research Program, Open University of Catalonia, Barcelona, Spain. ${ }^{6}$ Present address: Université de Paris, INCC UMR 8002, CNRS, F-75006 Paris, France. ${ }^{7}$ Present address: Institut Universitaire de France, Paris, France. ${ }^{8}$ These authors jointly supervised this work: Antoni Valero-Cabré and Marisa Carrasco. ${ }^{\varpi}$ email: yjlin@nyu.edu
} 
Neural entrainment-the progressive phase alignment of intrinsic oscillators by external sources of rhythmic stimulation (reviews ${ }^{35-37}$ ) - modulates behavioral performance in many visual ${ }^{38-44}$, tactile ${ }^{45}$, motor ${ }^{46}$, and memory ${ }^{47}$ tasks. Behavioral studies with concurrent TMS-EEG can shed light on neural oscillatory processes of behavior $^{43,44,46-48}$. Entrainment from higher-order to sensory areas has been proposed to underlie top-down modulations of attention ${ }^{40,42,43,49-52}$. Although visual signals in visuo-occipital areas are modulated by attention ${ }^{53-56}$, a fundamental question is whether and how the occipital cortex would respond to entrainment while participants perform a visual task, even without attention being explicitly manipulated. To address this question, we utilized a short-burst rhythmic TMS protocol along with concurrent EEG recordings ${ }^{43,57}$, targeting V1/V2 with visual field mapping by fMRI and/or phosphene induction.

For the frequency of alpha-band stimulation, previous short-burst TMS studies used either $10 \mathrm{~Hz}^{38,48}$ or individual alpha frequency (IAF) as the entrainment frequency ${ }^{57,58}$. IAF is a stable neurophysiological trait marker at rest ${ }^{59}$, which increases with task demand ${ }^{60,61}$ and reflects the task-required temporal integration window ${ }^{62}$. However, the assumption that matching the entraining frequency with IAF is important has not been directly tested in concurrent TMS-EEG studies ${ }^{37}$. Our strategy was to use $10 \mathrm{~Hz}$ as the entraining frequency and examine whether the degree of similarity between IAF and $10 \mathrm{~Hz}$ predicted the magnitude of phase-locked activities following entrainment.

Here we asked how the occipital cortex responds to entrainment while controlling spatial attention allocation to be distributed across the left and right visual fields, where the stimuli appeared. We aimed to entrain alphaband synchrony in early visual areas with rhythmic $10 \mathrm{~Hz}$ TMS and examine the neural signatures of entrainment with EEG. We hypothesized that the occipital alpha-band activity can be entrained by targeting V1/V2 because intrinsic alpha oscillators exist in $\mathrm{V} 1^{14,63}$ and $\mathrm{V} 2^{64}$. Given that V1/V2 play a pivotal role in early visual information processing, it is important to find out whether entrainment with rhythmic TMS can be established in these areas and how long such entrainment would last. We adopted arrhythmic-active TMS and rhythmic-sham stimulation control conditions, similar to previous TMS-EEG entrainment studies ${ }^{43,57}$. We systematically analyzed the temporal dynamics in multiple time windows during and after stimulation bursts. Although modulations of neural activity do not necessarily lead to changes in behavior, our secondary goal was to examine whether entrained alpha-band oscillations targeting early visual cortex (V1/V2) could affect perceptual discriminability and/or criteria in a visual discrimination task.

Based on our hypothesis that occipital alpha-band activity can be entrained by targeting V1/V2 with $10 \mathrm{~Hz}$ TMS bursts, we tested four specific predictions characterizing TMS-driven entrainment (the first three have been previously tested in frontal and parietal locations ${ }^{43,57}$ ): (1) progressive enhancement of entrainment: The strength of phase-locked activity, as measured by evoked (phase-locked) oscillation amplitude (as opposed to induced, non-phase-aligned, oscillation amplitude ${ }^{65}$ ) and ITPC (consistency of phase alignment) ${ }^{43,57}$, should be enhanced only after the second TMS pulse of the 4-pulse burst, because the alpha periodicity of the entraining rhythm is not defined until then. Otherwise, the results could simply reflect phase-resetting due to the first TMS pulse. (2) Output frequency specificity: Entrained phase-locked activity should peak around the 10-Hz TMS frequency. (3) Dependence on the intrinsic oscillation phase: Entrained activity should depend on the pre-TMS alpha phase ${ }^{57}$. Were the enhanced phase-locked activity due to reverberation of the imposed rhythm, rather than entrainment of intrinsic oscillators, the entrained activity should be similar regardless of pre-TMS alpha phase. (4) Input frequency specificity: The strength of the entrained activity should correlate with the degree of similarity between IAF and $10 \mathrm{~Hz}$, given that IAF may be the intrinsic oscillation frequency of occipital alpha oscillators ${ }^{57}$.

\section{Materials and methods}

Participants. All 11 participants (six male, five female; nine naïve to the purpose of the study; 20-47 years; $M=29.9 ; S D=6.8$ ) provided written informed consent. One participant's data were excluded due to excessive involuntary blinking. The number of participants was determined based on a power analysis $(\beta>0.8$ at alpha $=0.05$ ) from bootstrapping five participants' data, and was comparable to previous alpha-band entrainment TMS-EEG studies in the parietal cortex ${ }^{48,57}$. NYU institutional review board approved the protocol (IRB \#i14-00788), which followed the Declaration of Helsinki and safety guidelines for TMS experiments ${ }^{66}$.

Apparatus. The stimuli were presented on a ViewSonic P220f. monitor. The screen resolution was $800(\mathrm{H}) \times 600(\mathrm{~V})$ at $120 \mathrm{~Hz}$. The viewing distance was $57 \mathrm{~cm}$, set by a chin-rest. The stimuli presentation code was written in MATLAB with Psychtoolbox $3^{67,68}$. To linearize stimuli contrast, the monitor's gamma function was measured with a ColorCAL MKII Colorimeter (Cambridge Research Systems).

The TMS pulses were delivered with a 70-mm figure-of-eight coil controlled by a Magstim Super Rapid ${ }^{2}$ Plus $^{1}$ system. The coil, whose handle pointed rightward with respect to the midline of the skull, was supported by a mechanical arm and hand-held tangentially to the skull; its positioning was always guided by a Brainsight neuronavigation system (Rogue Research) with $\sim 1 \mathrm{~mm}$ precision. Two identical TMS coils were alternated every two blocks of testing to prevent overheating. Infrared reflecting markers were attached to the TMS coil and the participant's head to extract the relative $3 \mathrm{D}$ positions in real time; they were displayed on each participant's MRI structural scan.

The EEG system consisted of a Brain Products actiCHamp amplifier and TMS-compatible Easycap actiCAP slim caps. The EEG cap layout, following the international 10-20 system ${ }^{69}$, included a grid of 63 TMS compatible $6 \mathrm{~mm}$-thick electrodes, including a ground electrode placed at Fpz and a reference electrode placed at FCz. Impedance of all electrodes after conducting gel application was kept below $25 \mathrm{k} \mathrm{Ohm} \mathrm{during} \mathrm{the} \mathrm{whole} \mathrm{experi-}$ mental session. The EEG recording software was BrainVision Recorder. An in-house built TMS triggering and EEG event registering device with at least $0.4 \mathrm{~ms}$ resolution ensured timing precision at $2500 \mathrm{~Hz}$ EEG sampling rate $^{70}$ (Fig. S1A). 


\section{Experimental protocol}

A

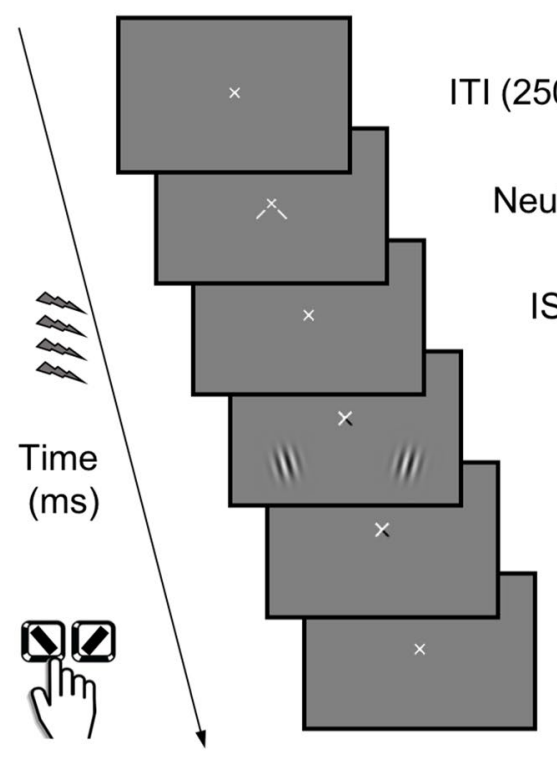

B

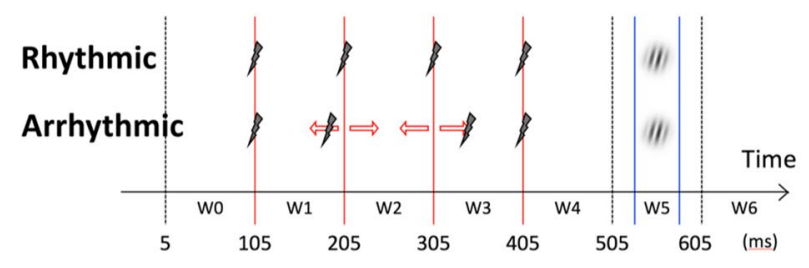

C

Gabors \& response cue $(50)$

Response cue only (575)

Response \& feedback

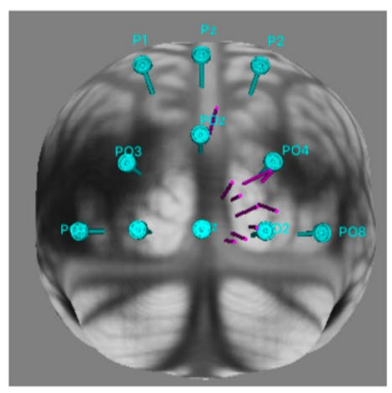

Figure 1. Experimental protocol (A) Trial structure. Each trial began with an intertrial period (ITI), followed by a neutral pre-cue to indicate the two stimulus locations. Active or sham stimulation was delivered in the interstimulus interval (ISI) between the neural pre-cue and the Gabor stimuli. The response cue indicated which Gabor was the target. Participants responded whether the target Gabor was tilted to the left or to the right with a key press. (B) Stimulation patterns and analysis time windows. The onset of the pre-cue is at $t=0$. The four red lines mark the pulse timings in the rhythmic condition. The two blue lines mark the timings of Gabors onset and offset. In the rhythmic condition, the gap between pulses was exactly $100 \mathrm{~ms}$. In the arrhythmic condition, the timing of the second and the third pulses was jittered (see text and Fig. S1 for the probability distribution). Time window $\mathrm{W} 0$ is the cycle before the first pulse. Time windows $\mathrm{W} 1, \mathrm{~W} 2$, and $\mathrm{W} 3$ are the cycles between pulse pairs. Time windows W4 and W5 are the first and the second cycles after the last pulse, respectively. (C) TMS loci and directions. Each cylinder represents the TMS directional vector of one participant on the MNI brain template. The cyan discs indicate the EEG electrode positions.

Visual discrimination task. With the goal of facilitating entrainment, we ensured that participants were engaged in a visual task. The participants performed an orientation discrimination task. Each trial began with a fixation period, followed by a $25 \mathrm{~ms}$ neutral pre-cue indicating that the target was equally likely to appear in the lower left or lower right visual field (Fig. 1A). After a $500 \mathrm{~ms}$ inter-stimulus interval (ISI), two Gabor patches and a response cue, indicating which patch was the target, appeared simultaneously. The Gabor patches (achromatic; $4 \mathrm{cpd} ; \sigma 0.42^{\circ}$ ) lasted $50 \mathrm{~ms}$. Participants were asked to indicate whether the target patch was slightly clockwise or counterclockwise relative to vertical via a key press (right index finger for the ' $\%$ ' key; left index finger for the ' $z$ ' key). A high pitch $(700 \mathrm{~Hz})$ signaled a correct response and a low tone $(400 \mathrm{~Hz})$ signaled an incorrect response. Only after the participants responded, the next trial would begin. The inter-trial interval was randomly chosen among 2500, 2750, or $3000 \mathrm{~ms}$.

A central fixation cross was constantly present. Participants were asked to maintain fixation at all times and to blink after each trial. An eye tracker (EyeLink1000) ensured that fixation was within a $1.5^{\circ}$ radius invisible circle. Trials for which fixation was broken (including blinking), from cue onset to stimuli offset, were discarded and repeated at the end of the block. The contrast level of the two Gabor patches were independently titrated before the experiment to attain a discrimination performance level of $\mathrm{d}^{\prime} \sim 1.2$. A method of constant stimuli (4-80\% Gabor contrasts in 7 log steps) was used to obtain the contrast at which sensitivity $\left(\mathrm{d}^{\prime}\right)^{71,72}$ reached half-of-max sensitivity of the Naka-Rushton function was defined as $c_{50}$ contrast. The group average (standard deviation) of $\mathrm{c}_{50}$ contrast was $16 \%$ (4\%) for both visual fields.

During the ISI, 4 rhythmic or arrhythmic (Fig. 1B), active or sham pulses were delivered. The ISI between the pre-cue and the first pulse was $80 \mathrm{~ms}$. The experiment applied a 2 (rhythmicity types) $\times 2$ (stimulation types) within-subject design. The rhythmic and arrhythmic stimulations were blocked. The stimulation type (active or sham) and the target side were randomized within blocks. The experiment consisted of eight blocks, each contained 64 trials, for a total of 512 trials; 128 trial repetitions per each of the four experimental conditions ( 2 rhythmicity types $\times 2$ stimulation types). Should we find differences between the rhythmic-active and arrhythmicactive contrast but not between the rhythmic-sham and arrhythmic-sham contrast, this design would allow us to rule out TMS sound, rhythmicity, and differences between rhythmically and arrhythmically interpolated EEG signals as plausible explanations of the entrainment effect.

Given that during TMS stimulation the participants were engaged in a visual discrimination task, as a secondary analysis, we assessed whether visual performance was affected by entrainment. There were no significant 
effects regarding whether entrainment modulates visual discrimination ${ }^{71,72}$, either for visual sensitivity ( $\mathrm{d}^{\prime}$; Fig. S2) or response criterion (c; Fig. S3). The error bars represent \pm 1 S.E.M. corrected for within-subject design ${ }^{73}$.

Transcranial magnetic stimulation. The TMS site for each participant was defined by retinotopy or phosphenes (see "Individualized TMS site"). Figure 1C illustrates the vectors connecting the cortical and scalp sites for each participant. All but one participant's stimulation vector clustered around electrodes $\mathrm{Oz}, \mathrm{O} 2$ and PO4. For that reason, we excluded that observer and replotted the data for the time frequency analysis. The TMS intensity was fixed at $70 \%$ for all participants (except for one at $65 \%$ and another at $67 \%$ ) of the maximal machine output, to ensure no phosphene induction during the task. The fixed upper bound of the TMS intensity was independently determined in pilot studies to ensure that the coil would not overheat in the middle of an experimental block. The sham control consisted of 4 pulses of pre-recorded TMS sounds played through a speaker attached to the coil. The coil was placed over the scalp, targeting the same area regardless of the actual stimulation type. Participants were asked to report if they saw any phosphenes at any point during the task; they reported none.

In the rhythmic condition, the pulses were $100 \mathrm{~ms}$ apart, aiming to induce alpha-band entrainment. In the arrhythmic condition, the timing of the first and last pulses of the burst were the same as in the rhythmic condition, whereas the timing of the second and the third pulses were randomly jittered on each trial according to a bimodal distribution synthesized from normal distributions $\mathrm{N}( \pm 30 \mathrm{~ms}, 10 \mathrm{~ms})$ (Fig. S1B). Before and after the experiment, we verified that the registered TMS timings on the EEG achieved the expected precision (Fig. S1C,D).

Individualized TMS site. We had magnetic resonance imaging (MRI) structural scan images and population receptive field (pRF) mapping ${ }^{74,75}$ data of the visual cortices for all but three participants. We used NiBabel ${ }^{76}$ to extract the right hemisphere V1 voxels corresponding to the location and size of the stimulus in the left visual field. The center of mass of the extracted voxels served as the individualized TMS target site. For the three participants without fMRI data, we used a TMS phosphene induction method ${ }^{56,77-81}$ to localize the optimal cortical site inducing unilateral phosphene perception in the left visual field (most likely on V1/V2 $2^{56,77,82-84}$ and probably $\mathrm{V}^{85,86}$ ). For these participants, the cortical site depth was arbitrarily set at $26.4 \mathrm{~mm}$, the average of the scalp-to-site distance of all other participants. Table S1 summarizes the TMS cortical and scalp sites in Montreal Neurological Institute (MNI) coordinates. At these individualized stimulation sites defined by pRF voxels, all but 1 participant reported seeing phosphenes; thus, the pRF and the phosphene induction method yielded comparable stimulation sites.

EEG preprocessing. The EEG recordings were digitized at $2500 \mathrm{~Hz}$ without any filtering. There was no re-referencing as the region of interest was the occipital cortex. The spike artifact caused by a TMS pulse typically lasted 4-10 ms. To reduce the artifact, signals within $1 \mathrm{~ms}$ before and $13 \mathrm{~ms}$ after each TMS pulse were replaced with shape-preserving piecewise cubic interpolated data (MATLAB command: interp1 with 'pchip' option $)^{43,48,57,87}$. The exact same procedure was applied to EEG data during sham auditory pulses to ensure that any statistical differences could not be attributed to discrepancies in data processing. No trials were discarded other than those with blinks (see above, "Visual discrimination task").

The continuous data were then down-sampled offline to $100 \mathrm{~Hz}$ and segmented into epochs containing data from $300 \mathrm{~ms}$ before to $900 \mathrm{~ms}$ after the neutral pre-cue onset. Preprocessing and analyses were carried out with MATLAB R2017a and software packages FieldTrip ${ }^{88}$ and Brainstorm ${ }^{89}$.

EEG analyses. Before the experiment, we recorded 2-min eyes-closed resting-state activity to define the individual alpha frequency (IAF) as the frequency corresponding to the maximum peak between 7 and $13 \mathrm{~Hz}$ on Welch's periodogram ${ }^{90}$ (MATLAB command: pwelch). To assess if the neural activity was phase-locked to the entraining periodic stimulation, we calculated two indices for each of the four conditions: (1) evoked oscillatory amplitude (square-root of power), by averaging waveforms across trials first and then applying Morlet wavelets $^{65} ;(2)$ inter-trial phase coherence (ITPC, or phase-locking value) ${ }^{91}$ by applying Morlet wavelets to each trial and calculating their consistency with $\left|\sum_{i=1}^{N} e^{i \theta} / N\right|$, where i denotes the trial number, $\mathrm{N}$ the total number of trials, and $\theta$ the phase. ITPC is a ratio between 0 and 1 , in which 1 indicates perfect phase alignment. For both indices, each Morlet wavelet had five cycles ${ }^{57}$, and the frequency range was 3-50 Hz. With five cycles of Morlet wavelets, the spectral bandwidth and wavelet duration, characterized by full width at half maximum, were $4.7 \mathrm{~Hz}$ and $0.2 \mathrm{~s}$, respectively. Thus, evoked oscillatory amplitude and ITPC centered around $10 \mathrm{~Hz}$ characterized the alpha-band $(8-12 \mathrm{~Hz}$ ) activity. Additionally, pre-TMS $\alpha$-phase was defined as the phase calculated from Morlet wavelets, $200 \mathrm{~ms}$ before the first TMS pulse ( $95 \mathrm{~ms}$ before the pre-cue onset).

We analyzed the evoked oscillation amplitude and ITPC in done planned time windows: W0 (5-105 ms, before the first pulse), W1 (105-205 ms, between the first and second pulses), W2 (205-305 ms, between the second and third pulses), W3 (305-405 ms, between the third and last pulses), W4 (405-505 ms, first cycle after the last pulse), W5 (505-605 ms, second cycle after the last pulse), and W6 (605-705 ms, third cycle after the last pulse). Because these time windows, which are consistent with reported time intervals of entrainment effects by TMS ${ }^{43,48,57}$, were specified a priori, multiple comparison correction was not required. But within each time window topographic analyses (paired t-tests between conditions across all channels) were corrected for multiple comparison with cluster-based permutation tests ${ }^{92}(1000$ permutations, one-tailed, alpha $=0.05$, cluster alpha $=0.05)$. According to the trial structure of the visual discrimination task, stimuli were presented in the middle of W5 (530-580 ms). Online eye tracking ensured that the trials containing eye blinking were discarded so that the analyzed EEG time windows were free of such artifacts. 


\section{Time-frequency analysis of phase-locked activities}

A

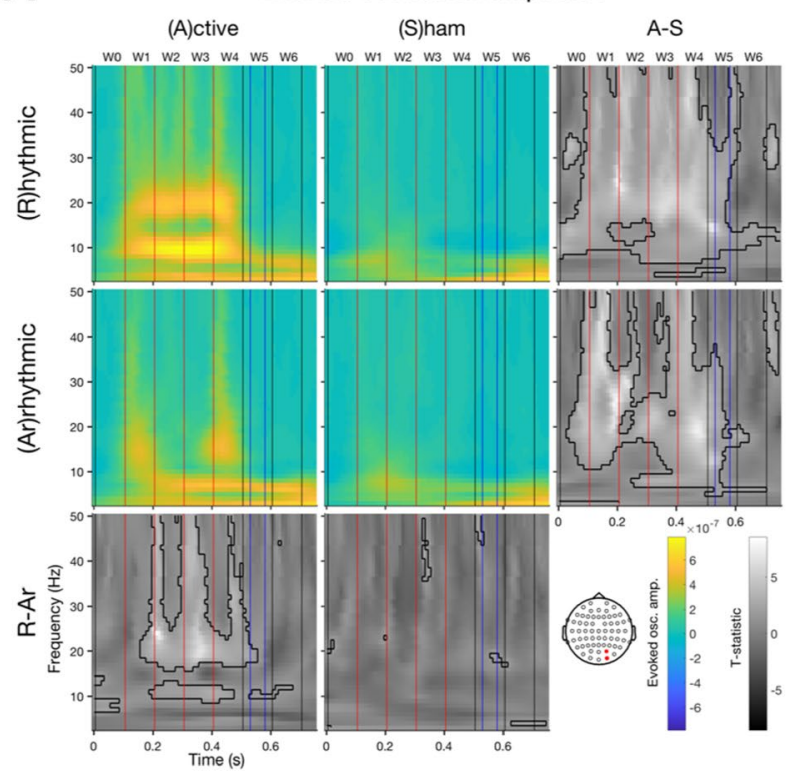

B

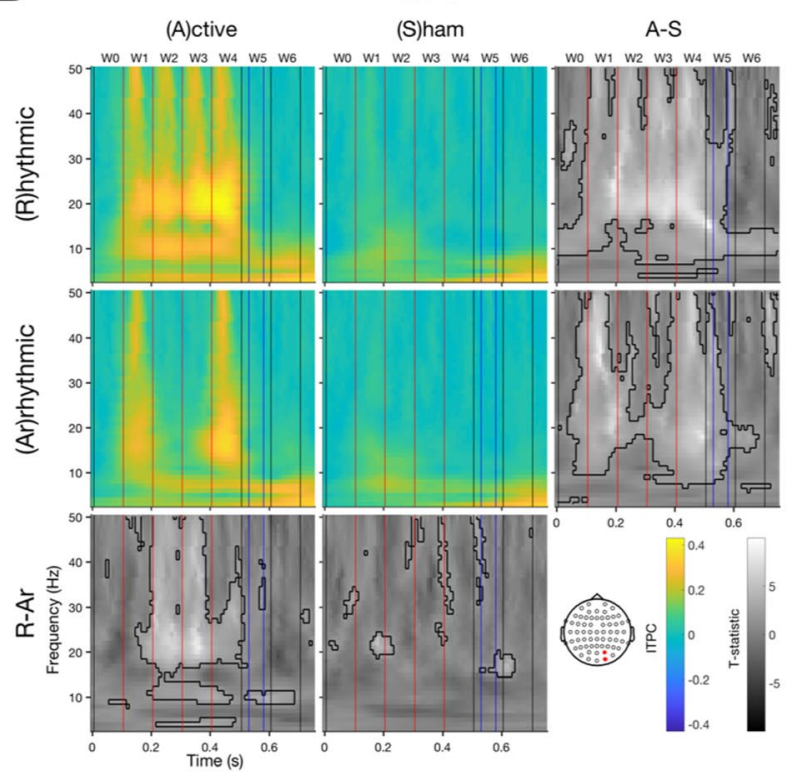

Figure 2. Time-frequency analysis of phase-locked activities. Colored panels are the group-averaged activities per condition (rhythmic vs. arrhythmic $\times$ active vs. sham stimulation). Gray panels are the $t$-statistics of condition contrasts per column or row. The pre-cue onset was defined as $t=0$. The electrode of interest is the average of the two channels (O2 \& PO4) near the TMS loci. In the t-statistic panels, the thick black contours indicate significant time-frequency bins. In each panel, the vertical black and red lines demarcate the $100 \mathrm{~ms}$ time windows, blue lines the Gabor onset and offset. The red lines also indicate the pulse timings in the rhythmic conditions. (A) Evoked oscillation amplitude. (B) ITPC. Each TMS pulse elicits broad band responses. Rhythmic-active stimulation elicits activities around $10 \mathrm{~Hz}$ and the first harmonic. Note that the arrhythmicactive condition is a more stringent control than the rhythmic-sham condition for the rhythmic-active condition. Note that at $10 \mathrm{~Hz}$, the full width at half maximum of time and frequency for Morlet wavelets with $\mathrm{c}=5$ are 0.19 and 4.71 , respectively.

Several statistical tests were performed. (1) As entrainment should lead to localized elevation of evoked oscillation amplitude and ITPC near the stimulation site, t-tests were performed by averaging the neural signatures in channels $\mathrm{O} 2$ and PO4 in the $10-\mathrm{Hz}$ band at all time windows. (2) To explore the temporal and spectral specificity of entrainment, $t$-tests of the two neural signatures were performed across all time-frequency bins averaged across channels $\mathrm{O} 2$ and PO4. (3) To explore the topography of neural activity before, during and after entrainment, t-tests were performed across all channels in the $10 \mathrm{~Hz}$ band at all time windows. (4) To examine whether ITPC enhancement following rhythmic TMS depended on pre-TMS alpha phase, trial-by-trial $10-\mathrm{Hz}$ phases $200 \mathrm{~ms}$ before the first pulse ( $95 \mathrm{~ms}$ before the pre-cue onset) were assorted into six equidistant phase bins. ITPC across participants and time points in W2-W6 indexed by these six phase bins were analyzed via one-way repeated measures ANOVA and a regression analysis with a sine wave $\left(y=a^{*} \sin \left(f^{*} x^{*} \pi / 3+\phi\right)+c\right.$, where $\mathrm{x}$ is the bin number; $\mathrm{a}, \mathrm{f}, \phi$, and $\mathrm{c}$ were free parameters $)^{57}$. (5) To examine the relation between IAF and ITPC, a linear regression was performed.

For the rhythmic-active condition, the arrhythmic-active condition is a more stringent control than the rhythmic-sham condition across all figures (Figs. 2, 3, 4, 5, S6-S9) (see also ${ }^{57}$ ). Across all time windows (Figs. 3, 4, 5, S6-S9), the contrasts between the rhythmic-active and arrhythmic-active conditions were significant whenever those between the rhythmic-active and rhythmic-sham condition were significant, except for evoked oscillation amplitude and ITPC in time window W0 (the $100 \mathrm{~ms}$ cycle before the first pulse) and evoked oscillation amplitude in time window W1 (the $100 \mathrm{~ms}$ cycle after the first pulse) (Figs. S6, S7A). Therefore, we report the statistical contrast between the rhythmic and arrhythmic-active stimulation conditions.

\section{Results}

We present psychophysics results, ERP analyses, wavelet analyses relevant for each of the four hypotheses, followed by exploratory analyses regarding the temporal dynamics of the topography of the entrainment effect. Altogether both sets of results indicate successful entrainment.

Psychophysics. Visual sensitivity ( $\mathrm{d}^{\prime}$ ) and criterion (c) in a discrimination task was unaffected by the elevated alpha-band evoked oscillation amplitude and ITPC (Figs. S2 and S3). 


\section{Topographic analysis of phase-locked $\alpha$-band activities in W4}

A

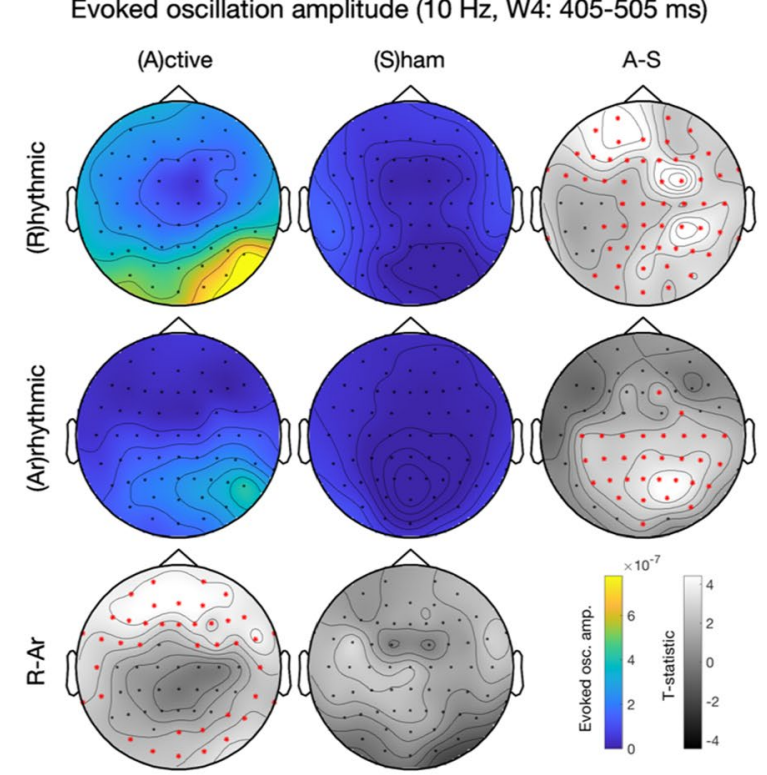

B

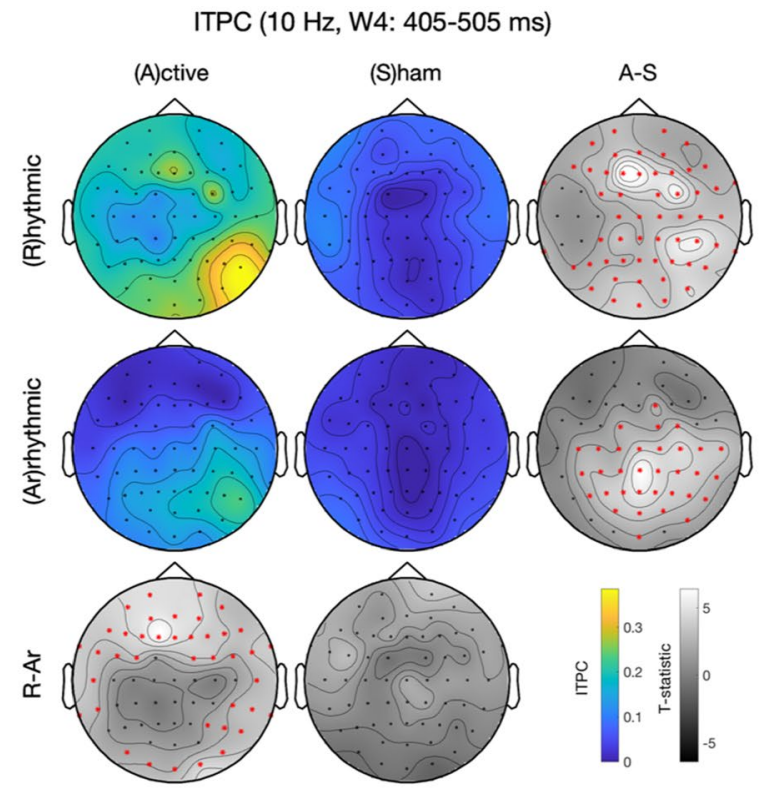

Figure 3. Topographic analysis of phase-locked $\alpha$-band activities in W4. The panel layout is similar to that in Fig. 2. The red star symbols indicate significant channels after cluster-based permutation test for multiple comparison correction. (A) Evoked oscillation amplitude. (B) ITPC. Rhythmic-active stimulation elicits widespread activation. With arrhythmic-active stimulation as control, there are significant clusters in the frontal and the occipital regions. Note that the patterns between $(\mathbf{A})$ and (B) are similar.

\section{Topographic analysis of phase-locked $\alpha$-band activities in W5}

A

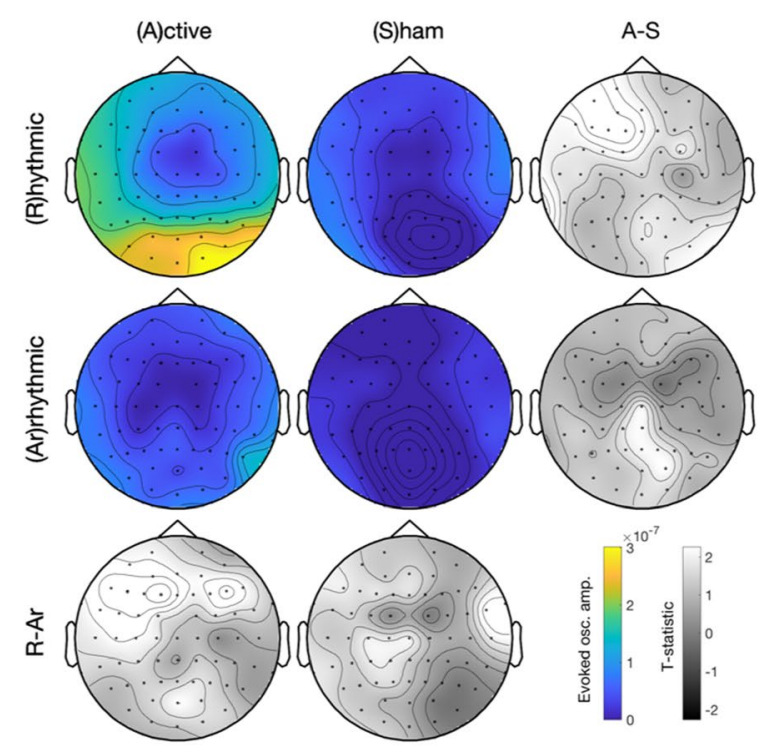

B

ITPC (10 Hz, W5: 505-605 ms)

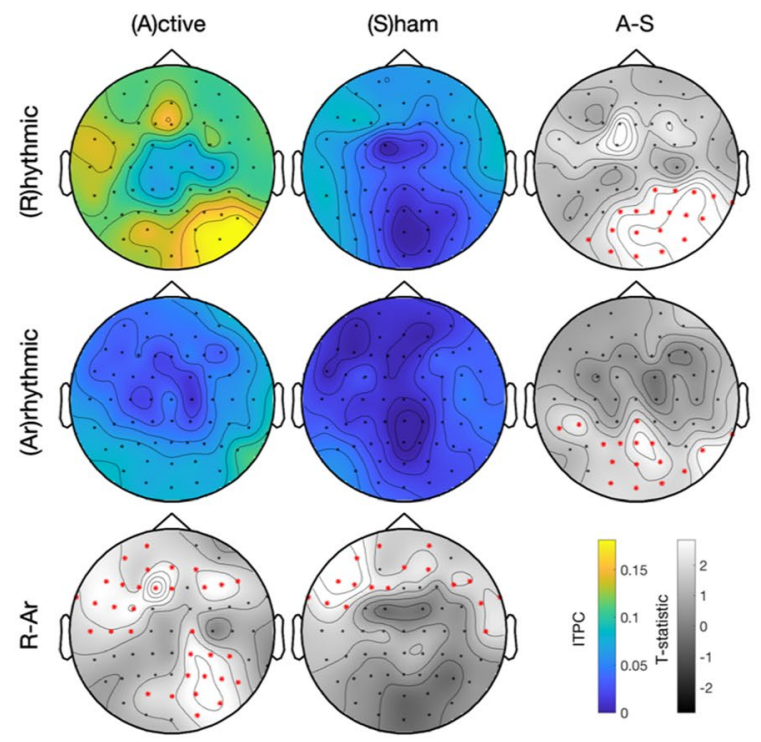

Figure 4. Topographic analysis of phase-locked $\alpha$-band activities in W5. See figure for panel layout description. (A) Evoked oscillation amplitude. (B) ITPC. Rhythmic-active stimulation elicits widespread activation. Note that (B) continues to show significant difference between conditions whereas (A) does not. 


\section{Topographic analysis of phase-locked $\alpha$-band activities in W6}

A

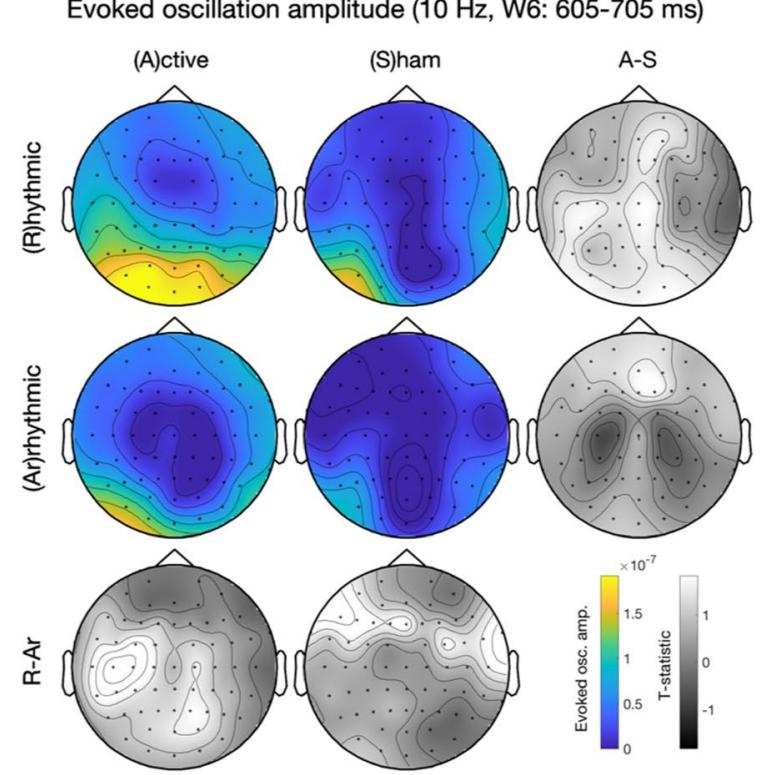

B

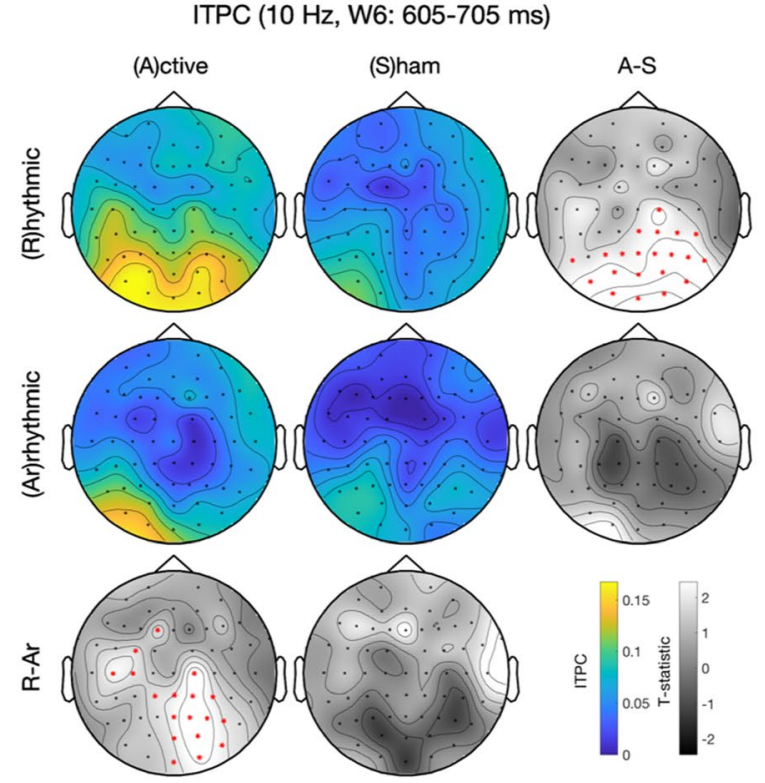

Figure 5. Topographic analysis of phase-locked $\alpha$-band activities in W6. See Fig. 4 for panel layout description. (A) Evoked oscillation amplitude. (B) ITPC. Rhythmic-active stimulation elicits widespread activation. Note that (B) continues to show significant difference between conditions whereas (A) does not. A significant lateralized parieto-occipital cluster exists in the rhythmic- vs. arrhythmic-active stimulation contrast.

\begin{tabular}{|l|l|l|l|l|l|l|l|}
\hline Time windows & W0 & W1 & W2 & W3 & W4 & W5 & W6 \\
\hline Evoked amplitude & 0.066 & 0.067 & $0.030^{*}$ & $0.015^{\star}$ & $0.021^{*}$ & $0.031^{\star}$ & 0.123 \\
\hline ITPC & 0.074 & 0.141 & $0.029^{*}$ & $0.004^{\star *}$ & $0.005^{\star *}$ & $0.010^{\star *}$ & $0.013^{\star}$ \\
\hline
\end{tabular}

Table 1. $p$ values of planned t-tests of evoked oscillation amplitude and ITPC at the $a$-band. The frequency of interest is $10 \mathrm{~Hz}$. The electrode of interest is the average of $\mathrm{O} 2$ and PO4 (near the stimulation loci; see Fig. 1C). The planned contrasts are between rhythmic and arrhythmic-active stimulation conditions in the seven predefined time windows, including the periods before (W0), during (W1-W3), and after (W4-W6) stimulation. The contrasts are significant starting from W2 until W5 or W6. ${ }^{*} \mathrm{p}<0.05 .{ }^{* *} \mathrm{p}<0.01$.

ERP. The spline interpolation method reasonably cleaned up the TMS spike artifacts present in the ERPs (Fig. S4). Apart from wavelet analyses, we carried out alpha-band bandpass filtering of ERPs across conditions (Fig. S5) and noted: (1) intrinsic alpha oscillations are evident before either active or sham stimulation, (2) in the active conditions, the pulse timings are closely aligned with alpha wave peaks, and (3) the intervals between waveform peaks are close to $100 \mathrm{~ms}$ only in the rhythmic-active condition.

Progressive enhancement of entrainment. To evaluate the temporal evolution of entrainment, we obtained t-test results of the planned contrasts between rhythmic and arrhythmic-active stimulation conditions in the seven pre-defined time windows ("Materials and methods") for evoked oscillation amplitude and ITPC (Table 1). Consistent with our prediction regarding entrained neural activity, the planned contrasts were not statistically significant 100-ms before and after the first TMS pulse (W0 and W1, $>>0.05$ ), but became significantly different for the later time windows (W2, W3, and $\mathrm{W} 4, \mathrm{p}<0.05$ ). Interestingly, the occipital alpha-band stimulation enhanced phase-locked activity during the second and the third cycles after the last pulse (significant contrasts in W5 and W6, p < 0.05). Our entrainment effect in terms of both evoked oscillation amplitude and ITPC lasted for 2-3 cycles ( 200 and $300 \mathrm{~ms}$, respectively, after the last pulse of the TMS burst).

Output frequency specificity of entrainment. To assess the output frequency-specificity, we conducted time-frequency analysis of phase-locked activities with frequencies ranging 3-50 Hz and time ranging 0-750 ms (Fig. 2). Rhythmic-active stimulation elicited activity at $\sim 10 \mathrm{~Hz}$ and its first harmonic $(\sim 20 \mathrm{~Hz})$. Additionally, there were broad band responses for each of the 4 TMS pulses. Contrasting the rhythmic-active condition with either the arrhythmic-active or the rhythmic-sham condition revealed that only the $\sim 10 \mathrm{~Hz}$ rather than the first harmonic component lasted beyond one cycle after the last TMS pulse, demonstrating output frequency specificity. Overall, the patterns for evoked oscillation amplitude and ITPC (Fig. 2A,B) were similar. 


\section{Regression analysis of ITPC}

A

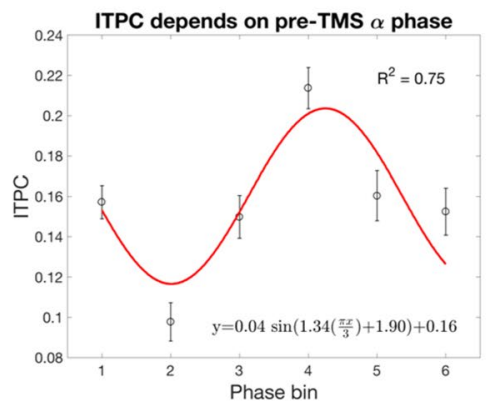

B

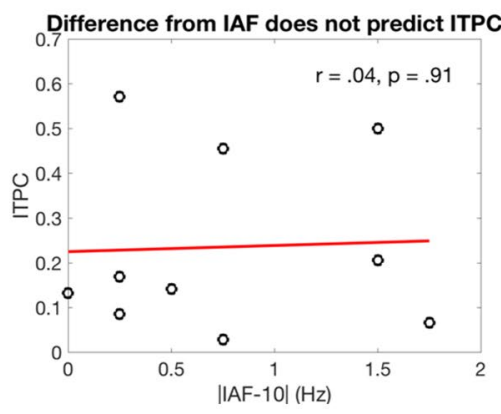

C

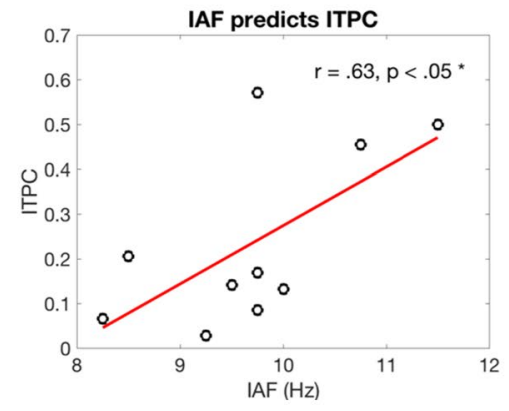

Figure 6. Regression analysis of ITPC in the rhythmic-active stimulation condition. The time window of ITPC is $0.205-0.705 \mathrm{~s}$, the same as the significant windows in Table 1. (A) ITPC depends on pre-TMS $\alpha$-phase ( $200 \mathrm{~ms}$ before the first pulse). Each point is the average of 510 samples ( 10 participants $\times 51$ time points). The error bars represent \pm 1 SEM. This $\sim 1$ period cyclic pattern suggests that the congruency between TMS and the phase of ongoing a oscillations determines the effectiveness of entrainment. Phase bin \#2 roughly corresponds to the $\sim 10 \mathrm{~Hz}$ ERP peak and phase bin \#5 to the trough. Note that due to conduction time the phase measured on the scalp does not indicate the exact phase of the oscillator in the brain. (B) Difference from IAF does not predict ITPC. The absolute difference between IAF and the entraining frequency does not predict ITPC during and after stimulation. (C) IAF predicts ITPC. IAF positively correlates with ITPC during and after stimulation. See text for discussion.

Dependence on the intrinsic oscillation phase. To ensure that the enhanced phase-locked activity reflected entrainment rather than reverberation of an externally imposed rhythm, we performed regression analyses on ITPC across the time windows that were significant (W2-W6) in Table 1. The pre-TMS phases (200 ms before the first $10-\mathrm{Hz}$ pulse) were assorted into 6 equidistant phase bins ${ }^{57}$. One-way repeated measures ANOVA revealed that the ITPC values across phase bins were significantly different $[F(9,45)=6.67, p<1 \mathrm{e}-5]$. In this period, ITPC depended on the phase bin with a sinusoidal trend (Fig. 6A), as previously reported ${ }^{57}$, suggesting that the phase of ongoing alpha oscillations matters. This result refutes the reverberation account, according to which ITPC would have been a flat line.

Input frequency dependence of entrainment. Inconsistent with the hypothesis that the intrinsic oscillators operate at the IAF in the occipital cortex, the degree of similarity between IAF and $10 \mathrm{~Hz}$ did not correlate with entrained ITPC $[r=0.04, p=0.91]$ (Fig. 6B). Instead, IAF directly correlated with entrained ITPC $[r=0.63$, $p<0.05$ ] (Fig. 6C). The degree of similarity between IAF and $10 \mathrm{~Hz}$ did not correlate with evoked oscillation amplitude $[r=0.15, p=0.68]$ (Fig. S10A). IAF did not correlate with evoked oscillation amplitude $[r=0.39$, $p=0.27$ ] (Fig. S10B). Moreover, pre-TMS total alpha oscillation amplitude did not predict entrained ITPC (Fig. S15).

Further analyses. Similar patterns of results were obtained for each of the previous analyses, when (a) the observer whose stimulation location (near POz) differed from the rest was excluded (Figs. S11, S12 and Table S2), and (b) taking only the trials that were preceded by correct responses (76\% of the data) (Figs. S13, S14 and Table S3). These results show that the effect generalizes across observers and that the feedback for incorrect responses did not drive the differential responses among the four conditions (rhythmic-sham, arrhythmic-sham, rhythmic-active and arrhythmic-active conditions).

Temporal dynamics of $\mathbf{1 0 - H z}$ topography. We explored the topography of the entrainment effects by performing topographic analysis of phase-locked alpha-band activities in each time window and assessing the temporal dynamics of evoked oscillation amplitude and ITPC. Cluster-based permutation tests ${ }^{92}$ were used for correction of multiple comparisons. Descriptively, with the same alpha threshold for cluster-based permutation test, $10-\mathrm{Hz}$ activity was initially widespread, likely due to EEG volume conduction, and progressively became more local over time. The statistically significant cluster included the frontal and occipital regions for both evoked oscillation amplitude and ITPC in W2-W4 (Figs. 3, S8, S9). Note that the occipital clusters in W2-W6 (Figs. 3, 4, 5, S8, S9) were more lateralized towards the stimulated (right) side.

A genuine entrainment effect should start in later time windows because this phenomenon has been characterized as a gradual process that realigns the intrinsic oscillator's phase (hence phase dependent, Fig. 6A) ${ }^{57}$. The hypothesis of progressive enhancement of entrainment was corroborated by the fact that the topographic contrast between rhythmic-active and arrhythmic-active stimulation was not significantly different for either evoked oscillation amplitude or ITPC in W0 (Fig. S6) and for evoked oscillation amplitude in W1 (Fig. S7). ITPC was significantly different during W1-W4 (Figs. 3, S7-S9) and continued to be so after evoked oscillation 


\begin{tabular}{|c|c|c|c|c|c|}
\hline Study & Stimulation site & Visual task & Frequency & \# pulses & \# cycles \\
\hline Current & Right V1/V2 (O2/PO4) & Discrimination & 10 & 4 & $\sim 3$ \\
\hline Vernet et al. $(2019)^{43}$ & Right FEF (FC2) & Detection & 30 & 4 & $\sim 1$ \\
\hline Thut et al. $(2011)^{57}$ & Right parietal (CP4) & N/A & IAF & 5 & $\sim 1$ \\
\hline Jaegle and Ro $(2014)^{48}$ & Right parietal (P2) & Discrimination & 10 & 3 & $\sim 1.5$ \\
\hline Jaegle and Ro $(2014)^{48}$ & Occipital $(\mathrm{Oz})$ & Discrimination & 10 & 3 & 0 \\
\hline
\end{tabular}

Table 2. Comparison with previous short-burst TMS-EEG entrainment studies. The stimulation site lists the target brain region along with the closet EEG electrode. The visual task is the task concurrent to TMS and control conditions. Most studies focus on the intrinsic alpha oscillations in the occipital or parietal cortices except one on the intrinsic beta oscillations in the frontal cortex. The number of cycles indicates how long the entrainment effect lasts following the last TMS pulse.

amplitude was no longer different in W5-W6 (Figs. 4, 5). This pattern of results based on topography paralleled the results from planned t-tests nearby the stimulation site (Table 1).

\section{Discussion}

This study is the first to show TMS entrainment of alpha activity in occipital cortex. We evaluated whether occipital alpha-band activity can be entrained by TMS targeting V1/V2 while participants performed a visual discrimination task. We tested the following hypotheses, based on established entrainment characteristics: (1) progressive enhancement of entrainment, (2) output frequency specificity, (3) dependence on the intrinsic oscillation phase, and (4) input frequency specificity. Our results are consistent with the first three hypotheses, revealing that occipital alpha activity can be entrained. However, the results were not consistent with the 4th hypothesis. Instead, IAF correlated with ITPC.

Successful and lasting alpha-band entrainment in the occipital cortex. With a 4-pulse 10-Hz TMS protocol, we successfully entrained the intrinsic alpha oscillations in the right occipital cortex for three cycles $(300 \mathrm{~ms})$ after the last TMS pulse. This novel result further supports that short-burst rhythmic TMS can effectively entrain frequency-specific neural synchrony, trial-by-trial, in the stimulated region. To our knowledge, this is the most effective short-burst TMS entrainment finding up to date. Previous TMS-EEG studies using a similar short-burst (3-5 pulses) rhythmic stimulation and control conditions achieved entrainment of intrinsic oscillations for about 1-1.5 cycles after the last pulse, with or without a concurrent visual task (Table 2). We may have obtained longer lasting TMS-entrained duration than the study entraining right IPS ${ }^{57}$ because our participants were engaged in a visual task, instead of in resting state. The only study evaluating TMS entrainment in occipital cortex reported not to find $\mathrm{it}^{48}$, even though their participants were also engaged in a visual discrimination task; their three 10-Hz TMS pulses may have been insufficient for entraining the occipital cortex. Future studies may further investigate which factors are crucial to successfully induce entrained synchronization of intrinsic neural oscillations outlasting rhythmic stimulation.

Note that our results fulfilled entrainment requirements and ruled out a reverberation account (as in ${ }^{57}$ ). Moreover, the evoked oscillation amplitude and ITPC at the alpha-band were not significantly different between the rhythmic and arrhythmic-active stimulation conditions in time windows W0 and W1. Therefore, it is unlikely that the lasting effects we found were due to temporal leakage of wavelets. The number of wavelet cycles $(c=5)$ in our study was the same as in the first concurrent TMS-EEG entrainment study ${ }^{57}$, where only $\sim 1$ cycle of alpha-band entrainment outlasting the last stimulation pulse was reported in posterior parietal region. Our design allowed us to rule out TMS sound, rhythmicity, and differences between rhythmically and arrhythmically interpolated EEG signals as plausible explanations of the entrainment effect.

Our results reveal that changes of two phase-locked activity measures-evoked oscillation amplitude and ITPC-show different temporal dynamics beyond the stimulation burst. ITPC lasted one more cycle than evoked oscillation amplitude (Table 1; Figs. 2, 4, 5). As ITPC is a phase-locking activity measure that does not take amplitude into account, this finding suggests that phase could be more sensitive and informative than amplitude to index the occurrence and duration of entrainment effects in the occipital cortex.

The finding that the occipital cortex can be entrained by TMS is consistent with the notion of inter-areal entrainment as a form of neural communication whose function could be to achieve local phase alignment ${ }^{36,49}$. Given that visual stimuli were presented at the same timing across all conditions, the continued phase-locking in the second and third cycles after the last pulse (W5 and W6) cannot be attributed to evoked alpha activity by target onset.

We note that during $10 \mathrm{~Hz}$ TMS (W1-W4) there were clear first harmonic $(20 \mathrm{~Hz})$ responses both in terms of evoked oscillation amplitude and ITPC (Fig. 2). However, after TMS (W5-W6) only the $10 \mathrm{~Hz}$ evoked oscillation amplitude and ITPC were long lasting. These lasting responses support the notion of entrainment. Furthermore, although our auditory sham did not produce a tactile stimulation, we note that this difference cannot explain the entrainment in the occipital cortices because a tactile entrainment would have been contralateral to TMS.

IAF predicts occipital entrainment. Some studies have assumed that using IAF, the intrinsic oscillation frequency of the parieto-occipital cortex at rest, as the entraining frequency would be optimal for entrainment, but this assumption has rarely been tested ${ }^{37}$. Similarly, our TMS-EEG findings are inconsistent with such an 
assumption: the dependency of ITPC on pre-TMS phase shows that entrainment effects depend on the ongoing oscillatory state while participants are engaged in a visual task, refuting a passive reverberation account (Fig. 6A). Therefore, intrinsic alpha oscillators exist in the occipital cortex. Furthermore, pre-TMS alpha power could not reliably predict entrained ITPC (Fig. S15). These findings are consistent with those of a transcranial alternating current stimulation (tACS)-EEG study ${ }^{93}$ showing that neither the baseline power nor IAF could reliably predict alpha power increase after $10-\mathrm{Hz}$ tACS entrainment.

The reason that the difference between the entraining frequency and IAF did not modulate ITPC (Fig. 6B) is likely the high stimulation intensity of TMS. Consistent with the idea of Arnold tongue (synchronization region defined on an amplitude-to-frequency plane) for oscillatory systems ${ }^{94}$, it has been shown that when the amplitude of the entraining stimulation is above a certain threshold, the output oscillating frequency is at a frequency equal to the stimulation instead of the intrinsic frequency ${ }^{95}$. With increasing intensity, a wider range of stimulation frequency centered around the intrinsic frequency could result in reliable entrainment as evidenced by the magnitude of phase-locking ${ }^{96}$.

The positive correlation between IAF and ITPC (Fig. 6C) suggests that individuals with higher IAF may be more susceptible to occipital alpha-band entrainment. A possible explanation is that the higher the IAF, the shorter the oscillation period. In response to external stimulation, shorter periods imply smaller absolute time steps in phase re-alignment. Future experimental studies may further exploit this interesting relation and examine if the same conclusion holds with IAF estimated during eyes-open resting state. This finding may also provide constraints for future cortical network modeling studies.

It is an open question whether the finding that IAF in the occipital cortex correlates with locally TMSentrained synchrony can be generalized to other brain areas, such as the parietal cortex or the somatosensory cortex. Interestingly, the closer the stimulation frequency to the individual beta frequency in the primary motor cortex, the more entrained the phase-locking ${ }^{46}$. Together with our findings, these results suggest that alpha and beta oscillations subserve distinct functions or that sensory and motor cortices have different operating principles.

Can alpha-band occipital TMS entrainment modulate visual sensitivity ( $\left.\mathbf{d}^{\prime}\right)$ ? Our results show that visual sensitivity and criterion in a discrimination task was unaffected by the elevated alpha-band evoked oscillation amplitude and ITPC (Figs. S2 and S3). Given that we only tested one specific target presentation timing, and at one TMS intensity per participant, our result does not necessarily rule out the possibility that changes in phase-locked activity level could modulate $\mathrm{d}^{\prime}$. For instance, a study showed that hit rate, but not false alarm rate, in the visual field ipsilateral to TMS was significantly higher immediately after a 5-pulse alpha-band TMS over the occipital cortex than that in the sham condition ${ }^{38}$. Likewise, after alpha-band visual flicker, discrimination accuracy oscillates $\sim 10 \mathrm{~Hz}^{97,98}$. Thus, the testing of multiple time lags at multiple intensities, as well as entraining other locations (e.g., electrode CP4), stimulating at different frequencies (e.g., theta), or manipulating spatial attention ${ }^{55,56,77}$ could inform whether accuracy oscillates after occipital TMS entrainment and whether more elevated evoked oscillation amplitude and ITPC are required for a significant behavioral outcome.

In contrast to phase-locked analyses, some visual detection studies with trial-by-trial spontaneous oscillation analysis have revealed that pre-stimulus alpha power ${ }^{99,100}$ and phase ${ }^{101}$ correlate with response criterion (c) instead of visual sensitivity $\left(\mathrm{d}^{\prime}\right)$. Further, for visual discrimination tasks, pre-stimulus alpha power correlates with confidence instead of accuracy ${ }^{102}$. However, note that without entrainment, trial-by-trial spontaneous prestimulus alpha activity is random and hence non-phase-locked across trials. Therefore, the current findings do not necessarily negate the possibility that entrainment may alter $\mathrm{d}^{\prime}$ following alpha-band entrainment.

Protocol considerations for future studies. An advantage of our protocol is that it has an arrhythmicactive stimulation control condition, similar to studies investigating IPS ${ }^{57}$ and FEF ${ }^{43}$ areas. Across time windows, using the arrhythmic-active and rhythmic-sham control conditions led to similar statistical outcomes; overall, the arrhythmic-active condition was a more stringent control condition resulting in less widespread significant statistical differences at the scalp level (Figs. 2, 3, 4, 5, S6-S9). Therefore, follow-up studies may consider removing the sham conditions to increase the statistical power of different types of TMS trials.

To further strengthen the importance of entraining near the intrinsic oscillation frequency, future studies could evaluate whether distinct TMS frequencies produce long lasting effects in other frequency bands. For instance, given our present findings establishing entrainment in the occipital cortex with TMS, the same protocol could be tailored to examine theta band $(4-7 \mathrm{~Hz})$ oscillations, which have also been reported during attention tasks ${ }^{77,103}$

Our 4-pulse alpha-band TMS protocol provides an interesting alternative for occipital entrainment to that of tACS protocols. Alpha-band tACS protocols typically involves $10-20 \mathrm{~min}$ of stimulation followed by $1-3 \mathrm{~min}$ of testing period, during which enhanced alpha-power or ITPC has been recorded with concurrent EEG and taken as evidence of entrainment ${ }^{93,104}$ (but see alternative interpretations ${ }^{105,106}$ ). In any case, TMS protocols ${ }^{48,57}$, including ours, provide more focal stimulation effects than tACS and can be effectively delivered in shorter 'bursts' of pulses, hence pinpoint more accurately the temporal dynamics of entrainment effects, enable trial-by-trial stimulation designs and concurrent EEG recordings.

Entrainment helps synchronization across neural populations and communication in the brain, and supports cognitive processes such as top-down attention ${ }^{40,42,43,49-52}$. External manipulation protocols via TMS can help establish the causal role of intrinsic oscillations within a cortical area on specific brain processes and behaviors, and their interactions with other brain regions. Regarding translational implications, the established causality could be conducive to identify abnormal rhythms and for potential interventions. Specific constraints discovered 
with one specific brain stimulation technology, such as TMS, may be also informative to other technologies, such as tACS.

To conclude, we have established a rhythmic 4-pulse alpha-band occipital TMS protocol for effective trialby-trial, online brain stimulation to enable alpha entrainment in retinotopically organized V1/V2 areas. With a 300 -ms entraining period, phase-locked activities persisted for $\sim 300 \mathrm{~ms}$ (three 10-Hz cycles) after the last pulse. To our knowledge, this is the longest lasting short-burst TMS entrainment finding reported up to date. Moreover, we found that IAF predicts the strength of entrained phase-locking across trials (ITPC). Therefore, IAF is a key factor worth investigating in future alpha entrainment studies.

Received: 30 May 2021; Accepted: 10 August 2021

Published online: 17 September 2021

\section{References}

1. Buzsáki, G. Rhythms of the Brain (Oxford University Press, 2006).

2. VanRullen, R. Perceptual cycles. Trends Cogn. Sci. 20, 723-735 (2016).

3. Dugué, L. \& VanRullen, R. Transcranial magnetic stimulation reveals intrinsic perceptual and attentional rhythms. Front. Neurosci. 11, 154 (2017).

4. Ergenoglu, T. et al. Alpha rhythm of the EEG modulates visual detection performance in humans. Cogn. Brain Res. 20, 376-383 (2004).

5. Hanslmayr, S. et al. Visual discrimination performance is related to decreased alpha amplitude but increased phase locking. Neurosci. Lett. 375, 64-68 (2005).

6. Hanslmayr, S. et al. Prestimulus oscillations predict visual perception performance between and within subjects. Neuroimage 37, 1465-1473 (2007).

7. Rahn, E. \& Başar, E. Enhancement of visual evoked potentials by stimulation during low prestimulus EEG stages. Int. J. Neurosci. 72, 123-136 (1993).

8. Romei, V. et al. Spontaneous fluctuations in posterior a-band EEG activity reflect variability in excitability of human visual areas. Cereb. Cortex 18, 2010-2018 (2008).

9. Romei, V., Rihs, T., Brodbeck, V. \& Thut, G. Resting electroencephalogram alpha-power over posterior sites indexes baseline visual cortex excitability. NeuroReport 19, 203-208 (2008).

10. Rahn, E. \& Başar, E. Prestimulus EEG-activity strongly influences the auditory evoked vertex response A new method for selective averaging. Int. J. Neurosci. 69, 207-220 (1993).

11. Ploner, M., Gross, J., Timmermann, L., Pollok, B. \& Schnitzler, A. Oscillatory activity reflects the excitability of the human somatosensory system. Neuroimage 32, 1231-1236 (2006).

12. Dugué, L., Marque, P. \& VanRullen, R. The phase of ongoing oscillations mediates the causal relation between brain excitation and visual perception. J. Neurosci. 31, 11889-11893 (2011).

13. Osipova, D., Hermes, D. \& Jensen, O. Gamma power is phase-locked to posterior alpha activity. PLoS ONE 3, e3990 (2008)

14. Dougherty, K., Cox, M. A., Ninomiya, T., Leopold, D. A. \& Maier, A. Ongoing alpha activity in V1 regulates visually driven spiking responses. Cereb. Cortex 27, 1113-1124 (2017).

15. Michalareas, G. et al. Alpha-beta and gamma rhythms subserve feedback and feedforward influences among human visual cortical areas. Neuron 89, 384-397 (2016).

16. van Kerkoerle, T. et al. Alpha and gamma oscillations characterize feedback and feedforward processing in monkey visual cortex. Proc. Natl. Acad. Sci. USA 111, 14332-14341 (2014).

17. Jensen, O. \& Mazaheri, A. Shaping functional architecture by oscillatory alpha activity Gating by inhibition. Front. Hum. Neurosci. 4, $186(2010)$.

18. Klimesch, W., Sauseng, P. \& Hanslmayr, S. EEG alpha oscillations the inhibition-timing hypothesis. Brain Res. Rev. 53, 63-88 (2007).

19. Palva, S. \& Palva, J. M. New vistas for a-frequency band oscillations. Trends Neurosci. 30, 150-158 (2007).

20. Kizuk, S. A. D. \& Mathewson, K. E. Power and phase of alpha oscillations reveal an interaction between spatial and temporal visual attention. J. Cogn. Neurosci. 29, 480-494 (2017).

21. Rohenkohl, G. \& Nobre, A. C. Alpha oscillations related to anticipatory attention follow temporal expectations. J. Neurosci. 31, 14076-14084 (2011).

22. Gould, I. C., Rushworth, M. F. \& Nobre, A. C. Indexing the graded allocation of visuospatial attention using anticipatory alpha oscillations. J. Neurophysiol. 105, 1318-1326 (2011).

23. Ikkai, A., Dandekar, S. \& Curtis, C. E. Lateralization in alpha-band oscillations predicts the locus and spatial distribution of attention. PLoS ONE 11, e0154796 (2016).

24. Kelly, S. P., Lalor, E. C., Reilly, R. B. \& Foxe, J. J. Increases in alpha oscillatory power reflect an active retinotopic mechanism for distracter suppression during sustained visuospatial attention. J. Neurophysiol. 95, 3844-3851 (2006).

25. Rihs, T. A., Michel, C. M. \& Thut, G. Mechanisms of selective inhibition in visual spatial attention are indexed by $\alpha-b a n d$ EEG synchronization. Eur. J. Neurosci. 25, 603-610 (2007).

26. Sauseng, P. et al. A shift of visual spatial attention is selectively associated with human EEG alpha activity. Eur. J. Neurosci. 22, 2917-2926 (2005).

27. Thut, G., Nietzel, A., Brandt, S. A. \& Pascual-Leone, A. $a$-band electroencephalographic activity over occipital cortex indexes visuospatial attention bias and predicts visual target detection. J. Neurosci. 26, 9494-9502 (2006).

28. Worden, M. S., Foxe, J. J., Wang, N. \& Simpson, G. V. Anticipatory biasing of visuospatial attention indexed by retinotopically specific a-band electroencephalography increases over occipital cortex. J. Neurosci. 20, RC63 (2000).

29. Yamagishi, N., Goda, N., Callan, D. E., Anderson, S. J. \& Kawato, M. Attentional shifts towards an expected visual target alter the level of alpha-band oscillatory activity in the human calcarine cortex. Cogn. Brain Res. 25, 799-809 (2005).

30. Yamagishi, N. et al. Attentional modulation of oscillatory activity in human visual cortex. Neuroimage 20, 98-113 (2003).

31. Capotosto, P., Babiloni, C., Romani, G. L. \& Corbetta, M. Frontoparietal cortex controls spatial attention through modulation of anticipatory alpha rhythms. J. Neurosci. 29, 5863-5872 (2009).

32. Capotosto, P., Babiloni, C., Romani, G. L. \& Corbetta, M. Differential contribution of right and left parietal cortex to the control of spatial attention A simultaneous EEG-rTMS study. Cereb. Cortex 22, 446-454 (2012).

33. Marshall, T. R., O’Shea, J., Jensen, O. \& Bergmann, T. O. Frontal eye fields control attentional modulation of alpha and gamma oscillations in contralateral occipitoparietal cortex. J. Neurosci. 35, 1638-1647 (2015).

34. Popov, T., Kastner, S. \& Jensen, O. FEF-controlled alpha delay activity precedes stimulus-induced gamma-band activity in visual cortex. J. Neurosci. 37, 4117-4127 (2017). 
35. Thut, G. et al. Guiding transcranial brain stimulation by EEG/MEG to interact with ongoing brain activity and associated functions A position paper. Clin. Neurophysiol. 128, 843-857 (2017).

36. Lakatos, P., Gross, J. \& Thut, G. A new unifying account of the roles of neuronal entrainment. Curr. Biol. 29, R890-R905 (2019).

37. Fröhlich, F. Experiments and models of cortical oscillations as a target for noninvasive brain stimulation. Prog. Brain Res. 222, 41-73 (2015).

38. Romei, V., Gross, J. \& Thut, G. On the role of prestimulus alpha rhythms over occipito-parietal areas in visual input regulation Correlation or causation?. J. Neurosci. 30, 8692-8697 (2010).

39. Romei, V., Thut, G., Mok, R. M., Schyns, P. G. \& Driver, J. Causal implication by rhythmic transcranial magnetic stimulation of alpha frequency in feature-based local vs. global attention. Eur. J. Neurosci. 35, 968-974 (2012).

40. Chanes, L., Quentin, R., Tallon-Baudry, C. \& Valero-Cabré, A. Causal frequency-specific contributions of frontal spatiotemporal patterns induced by non-invasive neurostimulation to human visual performance. J. Neurosci. 33, 5000-5005 (2013).

41. Quentin, R., Chanes, L., Vernet, M. \& Valero-Cabré, A. Fronto-parietal anatomical connections influence the modulation of conscious visual perception by high-beta frontal oscillatory activity. Cereb. Cortex 25, 2095-2101 (2015).

42. Quentin, R. et al. Visual contrast sensitivity improvement by right frontal high-beta activity is mediated by contrast gain mechanisms and influenced by fronto-parietal white matter microstructure. Cereb. Cortex 26, 2381-2390 (2016).

43. Vernet, M., Stengel, C., Quentin, R., Amengual, J. L. \& Valero-Cabré, A. Entrainment of local synchrony reveals a causal role for high-beta right frontal oscillations in human visual consciousness. Sci. Rep. 9, 14510 (2019).

44. Stengel, C., Vernet, M., Amengual, J. L. \& Valero-Cabré, A. Causal modulation of right hemisphere fronto-parietal phase synchrony with transcranial magnetic stimulation during a conscious visual detection task. Sci. Rep. 11, 3807 (2021).

45. Ruzzoli, M. \& Soto-Faraco, S. Alpha stimulation of the human parietal cortex attunes tactile perception to external space. Curr. Biol. 24, 329-332 (2014).

46. Romei, V. et al. Causal evidence that intrinsic beta-frequency is relevant for enhanced signal propagation in the motor system as shown through rhythmic TMS. Neuroimage 126, 120-130 (2016).

47. Hanslmayr, S., Matuschek, J. \& Fellner, M.-C. Entrainment of prefrontal beta oscillations induces an endogenous echo and impairs memory formation. Curr. Biol. 24, 904-909 (2014).

48. Jaegle, A. \& Ro, T. Direct control of visual perception with phase-specific modulation of posterior parietal cortex. J. Cogn. Neurosci. 26, 422-432 (2014).

49. Calderone, D. J., Lakatos, P., Butler, P. D. \& Castellanos, F. X. Entrainment of neural oscillations as a modifiable substrate of attention. Trends Cogn. Sci. 18, 300-309 (2014).

50. Lakatos, P., Karmos, G., Mehta, A. D., Ulbert, I. \& Schroeder, C. E. Entrainment of neuronal oscillations as a mechanism of attentional selection. Science 320, 110-113 (2008).

51. Fries, P. Rhythms for cognition communication through coherence. Neuron 88, 220-235 (2015).

52. Chanes, L., Chica, A. B., Quentin, R. \& Valero-Cabré, A. Manipulation of pre-target activity on the right frontal eye field enhances conscious visual perception in humans. PLoS ONE 7, e36232 (2012).

53. Beck, D. M. \& Kastner, S. Neural systems for spatial attention in the human brain: Evidence from neuroimaging in the framework of biased competition. In Nobre, A. C. \& Kastner, S. (Ed.) The Oxford Handbook of Attention (Oxford University Press, 2014).

54. Chica, A. B., Bartolomeo, P. \& Lupiáñez, J. Two cognitive and neural systems for endogenous and exogenous spatial attention. Behav. Brain Res. 237, 107-123 (2013).

55. Dugué, L., Merriam, E. P., Heeger, D. J. \& Carrasco, M. Differential impact of endogenous and exogenous attention on activity in human visual cortex. Sci. Rep. 10, 21274 (2020).

56. Fernández, A. \& Carrasco, M. Extinguishing exogenous attention via transcranial magnetic stimulation. Curr. Biol. 30, 1-7 (2020).

57. Thut, G. et al. Rhythmic TMS causes local entrainment of natural oscillatory signatures. Curr. Biol. 21, 1176-1185 (2011).

58. Klimesch, W., Sauseng, P. \& Gerloff, C. Enhancing cognitive performance with repetitive transcranial magnetic stimulation at human individual alpha frequency. Eur. J. Neurosci. 17, 1129-1133 (2003).

59. Grandy, T. H. et al. Peak individual alpha frequency qualifies as a stable neurophysiological trait marker in healthy younger and older adults. Psychophysiology 50, 570-582 (2013).

60. Haegens, S., Cousijn, H., Wallis, G., Harrison, P. J. \& Nobre, A. C. Inter- and intra-individual variability in alpha peak frequency. Neuroimage 92, 46-55 (2014).

61. Gray, M. J. \& Emmanouil, T. A. Individual alpha frequency increases during a task but is unchanged by alpha-band flicker. Psychophysiology 57, e13480 (2020).

62. Wutz, A., Melcher, D. \& Samaha, J. Frequency modulation of neural oscillations according to visual task demands. Proc. Natl. Acad. Sci. USA 115, 1346-1351 (2018).

63. Bollimunta, A., Mo, J., Schroeder, C. E. \& Ding, M. Neuronal mechanisms and attentional modulation of corticothalamic alpha oscillations. J. Neurosci. 31, 4935-4943 (2011).

64. Bollimunta, A., Chen, Y., Schroeder, C. E. \& Ding, M. Neuronal mechanisms of cortical alpha oscillations in awake-behaving macaques. J. Neurosci. 28, 9976-9988 (2008).

65. Tallon-Baudry, C. \& Bertrand, O. Oscillatory gamma activity in humans and its role in object representation. Trends Cogn. Sci. 3, 151-162 (1999).

66. Rossi, S., Hallett, M., Rossini, P. M., Pascual-Leone, A. \& of TMS Consensus Group, T. S. Safety, ethical considerations, and application guidelines for the use of transcranial magnetic stimulation in clinical practice and research. Clin. Neurophysiol. 120, 2008-2039 (2009).

67. Brainard, D. H. The psychophysics toolbox. Spat. Vis. 10, 433-436 (1997).

68. Pelli, D. G. The VideoToolbox software for visual psychophysics transforming numbers into movies. Spat. Vis. 10, 437-442 (1997).

69. Jasper, H. H. Report of the committee on methods of clinical examination in electroencephalography. Electroencephalogr. Clin. Neurophysiol. 10,370-375 (1958).

70. Lin, Y.-J. MarkStim: A TMS triggering/EEG event registering device (2013). https://github.com/mentist/markstim

71. Macmillan, N. A. \& Creelman, C. D. Detection Theory A User's Guide (Lawrence Erlbaum Associates, Inc., 2004),

72. Green, D. M. \& Swets, J. A. Signal Detection Theory and Psychophysics (John Wiley \& Sons, Inc., 1966).

73. Cousineau, D. Confidence intervals in within-subject designs A simpler solution to Loftus and Masson's method. Tutor. Quant. Methods Psychol. 1, 42-45 (2005)

74. Dumoulin, S. O. \& Wandell, B. A. Population receptive field estimates in human visual cortex. Neuroimage 39, 647-660 (2008).

75. Wandell, B. A. \& Winawer, J. Computational neuroimaging and population receptive fields. Trends Cogn. Sci. 19, 349-357 (2015).

76. Brett, M. et al. NiBabel: Access a cacophony of neuro-imaging file formats (2007). https://github.com/nipy/nibabel

77. Dugué, L., Roberts, M. \& Carrasco, M. Attention reorients periodically. Curr. Biol. 26, 1595-1601 (2016).

78. Schaeffner, L. F. \& Welchman, A. E. Mapping the visual brain areas susceptible to phosphene induction through brain stimulation. Exp. Brain Res. 235, 205-217 (2017).

79. Dugué, L., Marque, P. \& VanRullen, R. Transcranial magnetic stimulation reveals attentional feedback to area V1 during serial visual search. PLoS ONE 6, e19712 (2011). 
80. Dugué, L., Marque, P. \& VanRullen, R. Theta oscillations modulate attentional search performance periodically. J. Cogn. Neurosci. 27, 945-958 (2015).

81. Dugué, L., Beck, A.-A., Marque, P. \& VanRullen, R. Contribution of FEF to attentional periodicity during visual search A TMS study. eNeuro 6, ENEURO.0357-18.2019 (2019).

82. Salminen-Vaparanta, N. et al. Subjective characteristics of TMS-induced phosphenes originating in human V1 and V2. Cereb. Cortex https://doi.org/10.1093/cercor/bht131 (2013).

83. Salminen-Vaparanta, N., Noreika, V., Revonsuo, A., Koivisto, M. \& Vanni, S. Is selective primary visual cortex stimulation achievable with TMS?. Hum. Brain Mapp. 33, 652-665 (2012).

84. Silvanto, J., Muggleton, N., Lavie, N. \& Walsh, V. The perceptual and functional consequences of parietal top-down modulation on the visual cortex. Cereb. Cortex 19, 327-330 (2009).

85. Kastner, S., Demmer, I. \& Ziemann, U. Transient visual field defects induced by transcranial magnetic stimulation over human occipital pole. Exp. Brain Res. 118, 19-26 (1998).

86. Cowey, A. \& Walsh, V. Magnetically induced phosphenes in sighted, blind and blindsighted observers. NeuroReport 11, 3269$3273(2000)$.

87. Veniero, D. et al. Top-down control of visual cortex by the frontal eye fields through oscillatory realignment. Nat. Commun. 12, 1757 (2021).

88. Oostenveld, R., Fries, P., Maris, E. \& Schoffelen, J.-M. FieldTrip Open source software for advanced analysis of MEG, EEG, and invasive electrophysiological data. Comput. Intell. Neurosci. 2011, 156869 (2011).

89. Tadel, F., Baillet, S., Mosher, J. C., Pantazis, D. \& Leahy, R. M. Brainstorm A user-friendly application for MEG/EEG analysis. Comput. Intell. Neurosci. 2011, 879716 (2011).

90. Welch, P. D. The use of fast Fourier transform for the estimation of power spectra A method based on time averaging over short, modified periodograms. IEEE Trans. Audio Electroacoustics 15, 70-73 (1967).

91. Lachaux, J.-P., Rodriguez, E., Martinerie, J. \& Varela, F. J. Measuring phase synchrony in brain signals. Hum. Brain Mapp. 8, 194-208 (1999).

92. Maris, E. \& Oostenveld, R. Nonparametric statistical testing of EEG- and MEG-data. J. Neurosci. Methods 164, 177-190 (2007).

93. Helfrich, R. F. et al. Entrainment of brain oscillations by transcranial alternating current stimulation. Curr. Biol. 24, 333-339 (2014).

94. Pikovsky, A., Rosenblum, M. \& Kurths, J. Synchronization A Universal Concept in Nonlinear Sciences 52 (Cambridge University Press, 2001)

95. Herrmann, C. S., Murray, M. M., Ionta, S., Hutt, A. \& Lefebvre, J. Shaping intrinsic neural oscillations with periodic stimulation. J. Neurosci. 36, 5328-5337 (2016).

96. Notbohm, A., Kurths, J. \& Herrmann, C. S. Modification of brain oscillations via rhythmic light stimulation provides evidence for entrainment but not for superposition of event-related responses. Front. Hum. Neurosci. 10, 10 (2016).

97. de Graaf, T. A. et al. Alpha-band rhythms in visual task performance Phase-locking by rhythmic sensory stimulation. PLoS ONE 8, e60035 (2013).

98. Mathewson, K. E., Fabiani, M., Gratton, G., Beck, D. M. \& Lleras, A. Rescuing stimuli from invisibility inducing a momentary release from visual masking with pre-target entrainment. Cognition 115, 186-191 (2010).

99. Iemi, L., Chaumon, M., Crouzet, S. M. \& Busch, N. A. Spontaneous neural oscillations bias perception by modulating baseline excitability. J. Neurosci. 37, 807-819 (2017).

100. Limbach, K. \& Corballis, P. M. Prestimulus alpha power influences response criterion in a detection task. Psychophysiology 53, 1154-1164 (2016).

101. Sherman, M. T., Kanai, R., Seth, A. K. \& VanRullen, R. Rhythmic influence of top-down perceptual priors in the phase of prestimulus occipital alpha oscillations. J. Cogn. Neurosci. 28, 1318-1330 (2016).

102. Samaha, J., Iemi, L. \& Postle, B. R. Prestimulus alpha-band power biases visual discrimination confidence, but not accuracy. Conscious. Cogn. 54, 47-55 (2017).

103. Landau, A. N. \& Fries, P. Attention samples stimuli rhythmically. Curr. Biol. 22, 1000-1004 (2012).

104. Zaehle, T., Rach, S. \& Herrmann, C. S. Transcranial alternating current stimulation enhances individual alpha activity in human EEG. PLOS ONE 5, e13766 (2010).

105. Haberbosch, L. et al. Rebound or entrainment? The influence of alternating current stimulation on individual alpha. Front. Hum. Neurosci. 13, 43 (2019).

106. Vossen, A., Gross, J. \& Thut, G. Alpha power increase after transcranial alternating current stimulation at alpha frequency (a-tACS) reflects plastic changes rather than entrainment. Brain Stimulat. 8, 499-508 (2015).

\section{Acknowledgements}

This research was supported by National Institute of Health (NIH R21-EY026185 and R01-EY019693) to M.C., IHU-A-ICM-Translationnel, ANR projet Générique OSCILOSCOPUS and Flag-era-JTC-2017 CAUSALTOMICS to A.V.-C. We thank Antonio Fernández and Zhilin Zhang for assistance in data collection; Noah Benson and Marc Himmelberg for guidance on fMRI retinotopy data analysis; Antonio Fernández and Chloé Stengel for valuable discussions regarding this project; Rachel Denison and Florencia Assaneo for useful comments on the manuscript.

\section{Author contributions}

Y.-J.L., L.D., A.V.-C., and M.C. designed the study. Y.-J.L. and L.S. developed software and performed the experiment. Y.-J.L. analyzed data and prepared figures. Y.-J.L. wrote the manuscript (with guidance and supervision from M.C.). L.D., A.V.-C., and M.C. edited the manuscript. A.V.-C. and M.C. supervised the project. M.C. administered the project and acquired the funding.

\section{Competing interests}

The authors declare no competing interests.

\section{Additional information}

Supplementary Information The online version contains supplementary material available at https://doi.org/ 10.1038/s41598-021-96849-9.

Correspondence and requests for materials should be addressed to Y.-J.L.

Reprints and permissions information is available at www.nature.com/reprints. 
Publisher's note Springer Nature remains neutral with regard to jurisdictional claims in published maps and institutional affiliations.

(c) (i) Open Access This article is licensed under a Creative Commons Attribution 4.0 International License, which permits use, sharing, adaptation, distribution and reproduction in any medium or format, as long as you give appropriate credit to the original author(s) and the source, provide a link to the Creative Commons licence, and indicate if changes were made. The images or other third party material in this article are included in the article's Creative Commons licence, unless indicated otherwise in a credit line to the material. If material is not included in the article's Creative Commons licence and your intended use is not permitted by statutory regulation or exceeds the permitted use, you will need to obtain permission directly from the copyright holder. To view a copy of this licence, visit http://creativecommons.org/licenses/by/4.0/.

(C) The Author(s) 2021 